\title{
Carbon-Aware Business Process Design in Abnoba
}

\author{
Konstantin Hoesch-Klohe and Aditya Ghose \\ Decision Systems Lab (DSL), \\ School of Computer Science and Software Engineering, \\ University of Wollongong
}

\begin{abstract}
A key element of any approach to meeting the climate change challenge is the ability to improve operational efficiency in a pervasive fashion. The notion of a business process is a particularly useful unit of analysis in this context. This article describes a subset of the Abnoba framework for green business process management and shows how an algebraic framework can be leveraged to enable an environmental assessment on multiple heterogeneous dimensions (of qualitative or quantitative nature). Furthermore, a machinery for process improvement is outlined.
\end{abstract}

\section{Introduction}

The Abnoba framework enables business process management with explicit support for the environmental sustainability aspects of processes (represented in the Business Process Modeling Notation (BPMN) standard [1]). In particular we are concerned with business process design and re-design/improvement. This article extends our earlier work in the space 23. by describing a machinery for assessing the sustainability performance of a process design (which may be potentially incomplete) by aggregating task-level measures. These measures could include the carbon-footprint of a process (as assessed at design time), but also others, such as amount of water consumed, or the extent of waste generated. Our machinery uses an abstract algebraic framework (the c-semiring framework 4]) that enables assessment on multiple heterogeneous dimensions, which could be either qualitative or quantitative. The resulting framework extends the results of [5] and represents an improvement over other approaches to Quality of Service (QoS) modeling for processes [6 7. We further outline how sustainability-driven process improvement can be achieved by leveraging a library of process fragments. This machinery requires us to leverage the ProcessSEER framework for semantic effect annotation of process designs [8910].

\section{A Framework for Dealing with Green QoS Measures}

A key driver for process analysis, re-design and optimization is to satisfy or improve certain QoS measures. However, in practice QoS measures are often not commonly agreed upon and assessed on heterogeneous and multidimensional scales, which reduces their applicability (e.g. different machineries for each QoS measure have to 
be defined) [5]. We address these issues by deploying the algebraic c-semiring structure, which permits a multi-dimensional assessment of both qualitative and quantitative QoS factors. The framework generalizes many useful scales (real-valued, fuzzy-valued, probabilistic, qualitative and so on) and permits the integration of multiple heterogeneous scales into a single composite scale with the same abstract properties. Thus, while we might assess the carbon footprint of a process (or process step) on a real-valued scale, we might assess the impact of a process on flora/fauna on a qualitative scale of $\{$ high, medium, low $\}$. In this article we do not describe how to determine the values for the QoS measures, but note that they can be derived in a context specific manner, by correlating (rich) resource models with process designs (as described in our earlier work [3] ).

A c-semiring, which adds additional properties to the classic mathematical definition of a semiring, is defined as follows:

\section{Definition 1. (C-Semiring [4])}

$A$ semiring is a 5-tuple $\langle A, \oplus, \otimes, 0,1\rangle$ such that $A$ is a set of preference values, where $0,1 \in A$. 0 denotes the "worst" element and 1 denotes the "best" element in $A . \oplus$ is a cumulative, associative and idempotent comparison operator with 0 as identity element $(a \oplus 0=a)$ and 1 as absorbing element $(a \oplus 1=1)$, closed over $A$. $\otimes$ is an associative and commutative combination operator with 0 as its absorbing element $(a \otimes 0=0)$ and 1 as identity element $(a \otimes 1=a)$, closed over the set $A . \otimes$ distributes over $\oplus$.

The " $\oplus$ " comparing operator is used to define a partial order $\geq_{S}$ over the set of preference values $A$, enabling us to compare different elements of the semiring. On principle $a \geq_{S} b$ if and only if $a \oplus b=a$, denoting that the $\oplus$ operator choses $a$ over $b$. The $\otimes$ operator is used to combine elements of the set $A$. We have $a \otimes b \geq_{S} a(1$ is the maximum element of $A$ ), denoting that combining more elements of $A$ leads to a "worse" result with respect to $\geq_{S}$.

We require each activity to be annotated with its specific (local) QoS measures, represented by a vector $\left\langle m_{1}, m_{2}, \ldots, m_{k}\right\rangle$ where each $m_{i}$ is an element of $A$, of the csemiring associated with the $i$-th QoS measure. We accumulate QoS values across a process design, using the " $\otimes$ " combination operator, to receive the cumulative QoS values for each distinct QoS measure of the process design (see subsection 2.3 for more details).

\subsection{Green QoS Measures}

Our particular interest is in green QoS measures. In the following we list a (incomplete) list of green scales and illustrate their instantiation in the c-semiring structure. Other, QoS measures instantiated in the c-semiring structure can be found in $[5]$.

1. Water consumption: $\left\langle R^{+}, \min ,+, \infty_{+}, 0\right\rangle$

2. CO2-1 emission: $\left\langle R^{+}, \min ,+, \infty_{+}, 0\right\rangle$

\footnotetext{
${ }^{1}$ Carbon dioxide equivalent (CO2-e) is an expression of other greenhouse gases in their carbon dioxide equivalent by their global warming potential (CO2 itself has a global warming potential of 1 ).
} 
3. Waste generation: $\left\langle R^{+}, \min ,+, \infty_{+}, 0\right\rangle$

4. Damage to fauna and flora: $\langle\{l o w, \ldots$, high $\}$, min, $\max$, high, low $\rangle$

5. Air Quality : $\langle\{$ normal, ..., dangerous $\}$, min, max, dangerous, normal $\rangle$

6. Environmental performance: $\langle\{A A A, \ldots, D\}, \min , \max , D, A A A\rangle$

While "Water consumption", "CO2-e emission" and "Waste generation" are generally quantifiable, other measures like "damage to fauna and flora" are assessed in a qualitative scale, since their "real impact" can only be assessed in long term studies. Therefore, these values are often determined by an educated guesses of some expert. On the other hand "Air Quality" and "Environmental Performance" are qualitative measures, representing a combination of measures. In the next subsection we show how different heterogeneous c-semiring scales can be combined.

\subsection{Combining C-Semirings}

Each QoS scale is of individual interest. However, there is often the need to combine different measures to asses the overall environmental performance of a process design, considering all, or some subset of the green QoS scales. This results in a multidimensional QoS assessment. The resulting composite scale can be shown to have the same abstract properties (it is a c-semiring as well). More details and the corresponding proofs can be found in [4].

Definition 2. (Composition of C-Semirings) 4

Given $n$ semirings $S_{i}=\left\langle A_{i}, \oplus_{i}, \otimes_{i}, 0_{i}, 1_{i}\right\rangle$, for $i=1, \ldots, n$, let us define the structure $\operatorname{Comp}\left(S_{1}, \ldots, S_{n}\right)=\left\langle\left\langle A_{1}, \ldots, A_{n}\right\rangle, \oplus, \otimes,\left\langle 0_{1}, \ldots, 0_{n}\right\rangle,\left\langle 1_{1}, \ldots, 1_{n}\right\rangle\right\rangle$. Given $\left\langle a_{1}, \ldots, a_{n}\right\rangle$ and $\left\langle b_{1}, \ldots, b_{n}\right\rangle$ such that $a_{i}, b_{i} \in A_{i}$ for $i=1, \ldots, n$, $\left\langle a_{1}, \ldots, a_{n}\right\rangle \oplus\left\langle b_{1}, \ldots, b_{n}\right\rangle=\left\langle a_{1} \oplus_{1} b_{1}, \ldots, a_{n} \oplus_{n} b_{n}\right\rangle$, and $\left\langle a_{1}, \ldots, a_{n}\right\rangle \otimes\left\langle b_{1}, \ldots, b_{n}\right\rangle=$ $\left\langle a_{1} \otimes_{1} b_{1}, \ldots, a_{n} \otimes_{n} b_{n}\right\rangle$.

Accordingly, the order $\geq_{S}$ over $\left\langle A_{1}, \ldots, A_{n}\right\rangle$ is $\left\langle a_{1}, \ldots, a_{n}\right\rangle \geq_{S}\left\langle b_{1}, \ldots, b_{n}\right\rangle$ if and only if $\left\langle a_{1}+{ }_{1} b_{1}, \ldots, a_{n}+{ }_{n} b_{n}\right\rangle=\left\langle b_{1}, \ldots, b_{n}\right\rangle$. Since we have only defined the least upper bound $\left(\left\langle 0_{1}, \ldots, 0_{n}\right\rangle\right)$ and greatest lower bound $\left(\left\langle 1_{1}, \ldots, 1_{n}\right\rangle\right)$ not all elements of the composed c-semiring structure can be compared or combined. For example, consider the following composition of c-semiring instantiations for CO2-e and damage to fauna and flora. $\left\langle\left\langle R^{+},\{l, m, h\}\right\rangle, \oplus, \otimes,\left\langle\infty_{+}, h\right\rangle,\langle 0, l\rangle\right\rangle$. Let us first compare the elements $\langle 5, m\rangle \oplus\langle 4, l\rangle=\langle 4, l\rangle$, hence $\langle 4, l\rangle \geq_{S}\langle 5, m\rangle(a+b=a)$. However, $\langle 5, l\rangle \oplus\langle 4, h\rangle=\langle 4, l\rangle(a+b=c)$ is not comparable, since there is no order for these two tuples. We can solve this by defining a new order $\geq_{S}^{\prime}$ over each tuple in $\left\langle A_{1}, \ldots, A_{n}\right\rangle$ such that there does not exist a situation where $a \geq_{S} b$ and $a \neq b$ and $b \geq_{S}^{\prime} a$ (the orders contradict each other).

On the other hand, we can map each tuple of the combined c-semiring into a set $A$ of another c-semiring and define the order over this new structure. For example, scales for "fine particles in the air", "ground-level ozone", and "carbon monoxide" (besides others) can be composed and the resulting composition be mapped into the "Air Quality" c-semiring. Such a mapping is particularly appealing when a combination of different scales have to be communicated to stakeholders like the government, business partners or costumers. Similar, the measure "Environmental 
performance" $\langle\{A A A, \ldots, D\}, \min , \max , D, A A A\rangle$, listed in subsection 2.1, subsumes other environmental scales for ease of communication, where $A A A$ denotes the "best" environmental performance and $D$ the "worst" (similar scales are used to rate credits in the financial sector).

\subsection{QoS Measure Accumulation}

In this subsection we show how the QoS values (annotated at each activity) for distinct QoS measures can be accumulated across a process design to receive cumulative QoS values for each path through the process design.

The task of accumulating QoS measures across business process designs is not trivial since there might be various paths that can be traversed during process execution. We refer to each path through the process model, starting from a "start event" to a (user) selected activity, as a scenario label. A scenario label consists of a sequence $(\langle\rangle)$ or a set $(\{\})$ or a combination of both. Sets can be processed in any order and are used to represent parallel splits, while the sequence dictates an order to account for the sequence in which the activities of a design are modeled.

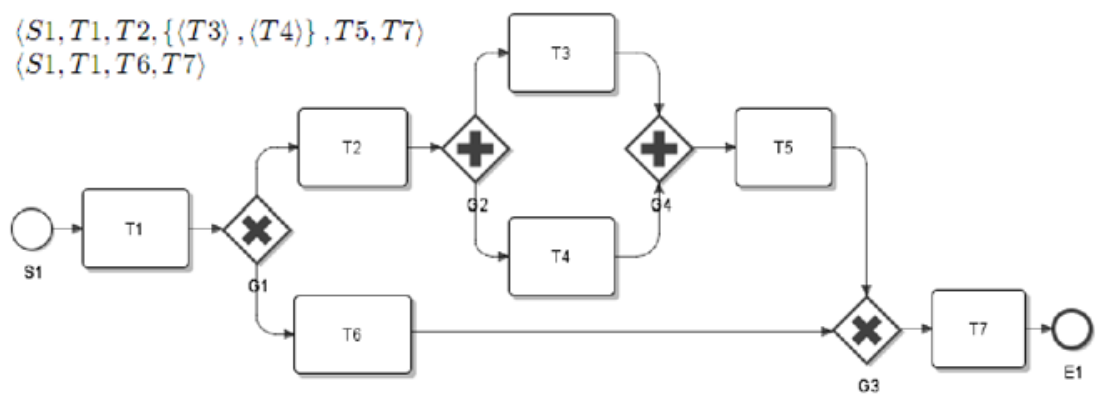

Fig. 1. BPMN Process Design

Figure1shows a BPMN process design with an exclusive split after activity $T 1$ denoting that during process execution either the "upper path" $(T 2, T 3, T 4, T 5)$ or the "lower path" (T6) can be taken, before both scenarios continue with activity $T 7$ and terminate at $E 1$. The corresponding scenario labels for selecting activity $T 7$ are shown at the upper left corner of the Figure1.

We accumulate as follows. Let $S$ be a set of c-semiring, each denoting a distinct QoS measure for a given process design. We accumulate annotated QoS values, where each value corresponds to an element of the set $A$ of each $s \in S$, between a pair of contiguous activities $T i$ and $T j$ ( $T i$ pointing to $T j$ ) as follows. The (cumulative) QoS values of a measure $s \in S$ at $T j$ consists of $a_{i}^{c u}(s) \otimes_{s} a_{j}^{l o}(s)$, where $a_{i}^{c u}(s)$ is a cumulative QoS value associated with $T i$ and $a_{j}^{l o}(s)$ is a local QoS value of $T j$. The operator ' $\otimes_{s}$ ' is the corresponding combination operator for c-semiring $s \in S$. We deal with XOR merges (see Figure 1label G3) in the following manner. If $T i$ and $T j$ are the only two activities immediately preceding an XOR-merge, and 
$T m$ is the activity immediately following it, we proceed by $a_{i}^{c u}(s) \otimes a_{m}^{l o}(s)=a_{m}^{c u}(s)$ and $a_{j}^{c u}(s) \otimes_{s} a_{m}^{l o}(s)=a_{m}^{c u}(s)^{\prime}$. To deal with AND merges (see Figure11abel $G_{4}$ ) we have to add an additional parallel combination operator " $\circledast$ " to the semiring structure. This operator allows us to specify how the QoS measures are propagated along a parallel branch and combined together at the merge gateway. For example, such an operator is needed when dealing with cycle time, where the "worst" time value of each branch in a parallel environment is picked. Let $T i$ and $T j$ be the only two activities immediately preceding an AND-merge, and $T m$ is the activity immediately following it, we proceed by $\left(a_{i}^{c u}(s) \circledast_{s} a_{j}^{c u}(s)\right) \otimes_{s} a_{m}^{l o}(s)=a_{m}^{c u}(s)$. In the case of cycle time, the $\circledast$ operator (choose max value) is idempotent, therefore $\otimes$ distributes over $\circledast$.

\section{Green Process Improvement}

In this section we outline (space constraints do not permit a more detailed elaboration) a machinery for identifying process re-designs with a more desired sustainability profile and the same functional outcome as the original design, using a library of process fragments. A process fragment is a (sub)process graph with a single entry and exit point (using process fragments any composition of well formed fragments results in a well formed process design).

First we require the activities of a process design to be annotated with semantic effects (denoting the post-conditions of the individual activity). These semantic effect annotations are represented in conjunctive normal form (CNF) and are accumulated along the execution paths of a process design (as described in 89. and implemented in the ProcessSEER [10] tool), to obtain the cumulative effects at the end-event of the process design. The final cumulative effects (representing intended effects ) determine functional requirements for the re-designed processes. Consequently, any process re-design has to meet these the functional requirements.

Second, we define a library of (semantic annotated) process fragments. These fragments can denote single activities, processes, or potentially services derived from a service broker. Essentially, we enable the replacement of fragments of a process design with other fragments drawn from the library in an (exhaustive search) procedure that ensures that the original functionality of the process is still realized, but the sustainability profile of the resulting process design is improved.

Third, in the context of process improvement, there is often a requirement of minimally change existing process designs, i.e., maximizing process improvement while minimizing disruption to the status quo. This is particularly important if we are interested in protecting investment put in existing process infrastructure and minimizing the ancillary costs associated with any change to process designs. The requirement for minimal change could be dealt with by design proximity as a tie breaker when multiple alternative process re-designs achieve the same quantum of process improvement. Instances of proximity relations and measures can be found in [8] and [1]. 


\section{Conclusion}

In this article we described a subset of the Abnoba framework for green business process management. In this context we first showed how the abstract algebraic c-semiring structure can be leveraged to deal with heterogeneous and multidimensional green measures. We further sketched a machinery for green business process improvement. A first evaluation with a handcrafted library, confirms the soundness of the approach, but its applicability in an industry setting remains future work.

\section{References}

1. Object Management Group: Business process modeling notation (bpmn) 1.2. , (2009), http://www .omg.org/spec/BPMN/1.2/PDF last checked 21.02

2. Ghose, A., Hoesch-Klohe, K., Hinsche, L., Le, L.S.: Green business process management: A research agenda. Australian Journal of Information Systems 16 (2009)

3. Hoesch-Klohe, K., Ghose, A.: Towards Green Business Process Management. In: Proc. of the 7th International Conference on Services Computing (Industry and Application Track) (2010)

4. Bistarelli, S., Montanari, U., Rossi, F.: Semiring-based constraint satisfaction and optimization. Journal of the ACM (JACM) 44(2), 236 (1997)

5. Ghose, A., Koliadis, G.: Pctk: A toolkit for managing business process compliance. In: Proc. of the Second International Workshop on Juris-informatics (2008)

6. Pavlovski, C., Zou, J.: Non-functional requirements in business process modeling. In: Proc. of the fifth on Asia-Pacific conference on conceptual modelling (2008)

7. Gorton, S., Reiff-Marganiec, S.: Towards a task-oriented, policy-driven business requirements specification for web services. In: Dustdar, S., Fiadeiro, J.L., Sheth, A.P. (eds.) BPM 2006. LNCS, vol. 4102, p. 465. Springer, Heidelberg (2006)

8. Ghose, A., Koliadis, G.: Auditing business process compliance. In: Krämer, B.J., Lin, K.-J., Narasimhan, P. (eds.) ICSOC 2007. LNCS, vol. 4749, pp. 169-180. Springer, Heidelberg (2007)

9. Ghose, A., Koliadis, G.: Pctk: A toolkit for managing business process compliance. In: Proc. of the 2008 International Workshop on Juris Informatics (2008)

10. Hinge, K., Ghose, A., Koliadis, G.: Process seer: A tool for semantic effect annotation of business process models. In: Proc. of the 13th IEEE International EDOC Conference, EDOC 2009 (2009)

11. Morrison, E.D., Menzies, A., Koliadis, G., Ghose, A.K.: Business process integration: Method and analysis. In: Proc. Sixth Asia-Pacific Conference on Conceptual Modelling, APCCM 2009 (2009) 